\title{
An expeditious synthesis of novel pyranopyridine derivatives involving chromenes under controlled microwave irradiation
}

\author{
Dushyant Singh Raghuvanshi and Krishna Nand Singh* \\ Department of Chemistry, Faculty of Science,Banaras Hindu University, \\ Varanasi -221005, India \\ E-mail: knsinghbhu@yahoo.co.in
}

\begin{abstract}
An efficient synthesis of novel pyrano[2,3-b]pyridine derivatives has been achieved by the cyclocondensation of 2-amino-3-cyano- $4 H$-chromenes and cyclohexanone in the presence of aluminium chloride under controlled microwave irradiation. The experimental conditions have been thoroughly optimized and established, allowing significant rate enhancements and excellent yields. The starting $4 H$-chromenes were obtained using one pot DBU-catalysed microwave induced multicomponent condensation of resorcinol, malononitrile and aromatic aldehydes.
\end{abstract}

Keywords: Friedländer annulation, pyranopyridines, microwave synthesis, multi-component reaction, 2-amino-3-cyano-4H-chromenes

\section{Introduction}

Pyranopyridines constitute an important class of heterocyclic compounds having diverse biological activities such as anti-allergic, anti-inflammatory, and estrogenic. ${ }^{1,2}$ Among the different substitution patterns that are known, benzopyrano[2,3-b]pyridines exhibit antiproliferative, ${ }^{3}$ cancer chemopreventive, ${ }^{4}$ anti-bacterial (including anti-tubercular), ${ }^{5}$ antimyopic, ${ }^{6}$ anti-histamic, ${ }^{7}$ hypotensive, ${ }^{8}$ anti-rheumatic, ${ }^{9}$ and antiasthmatic activities. ${ }^{10}$ Chromenes are important oxygenated heterocyclic compounds endowed with activities such as antidepressant, antihypertensive, anti-tubulin, antiviral, antioxidative, activator of potassium channels and inhibition of phosphodiesterase IV or dihydrofolate reductase, etc. ${ }^{11-21}$ As a result, a number of methodologies have been developed to synthesize chromene compounds. ${ }^{22-27}$

Pyranopyridines have become the object of current synthetic developments and many strategies for their synthesis have been adopted. ${ }^{28}$ The most simple and straightforward approach for the synthesis of polysubstituted pyridines and related azaheterocycles happens to be the Friedländer annulation. Among various Friedländer syntheses, cyclocondensation of oaminobenzonitrile with various ketones has been less explored. Previous investigations ${ }^{29,30}$ on 
condensation of these derivatives required prolonged reaction times, use of hazardous reagents and low yields. In view of the above, the development of an efficient and convenient protocol for the synthesis of pyranopyridines is of considerable interest.

Multicomponent reactions (MCRs) have recently emerged as valuable tool in the preparation of structurally diverse chemical libraries of drug-like heterocyclic compounds. ${ }^{31}$ There is great current interest in microwave assisted organic synthesis (MAOS), ${ }^{32}$ because such environmentally benign chemical methodologies are strongly required in light of the paradigm shift to "Green Chemistry". According to the current synthetic requirements, environmentally benign multi-component procedures employing microwave (MW) methodology are particularly welcome due to their intrinsic advantages. ${ }^{33}$

In view of the above, a facile, fast and high yielding synthesis of novel 11-amino-12-aryl7,9,10,12-tetrahydro-8H-5-oxa-6-aza-naphthacen-3-ols 6a-h has been achieved by the cyclocondensation of 2-amino-3-cyano-7-hydroxy-4-aryl-4H-chromenes $\mathbf{4 a - h}$ with cyclohexanone in the presence of $\mathrm{AlCl}_{3}$ under controlled $\mathrm{MW}$ irradiation. The substrates 2amino-3-cyano-4H-chromenes 4 a-I have been accomplished by the multicomponent reaction of resorcinol, malononitrile, and aromatic aldehydes using controlled MW irradiation in the presence of catalytic amount of DBU $(5 \mathrm{~mol} \%)$ in ethanol at an ambient temperature $50^{\circ} \mathrm{C}$. (Scheme 1).

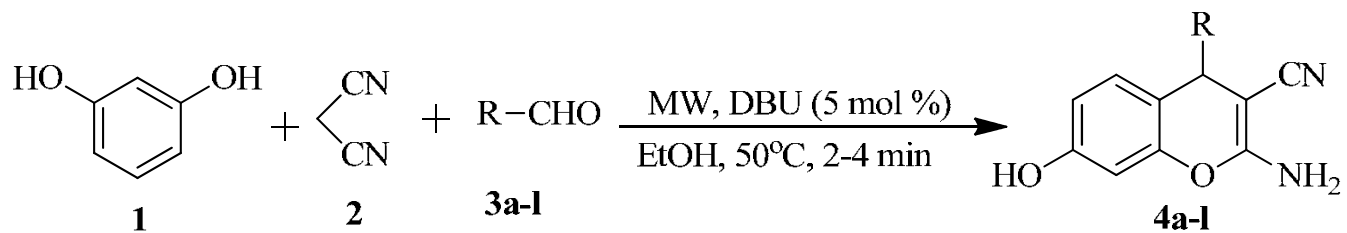

$\mathrm{R}=\mathrm{Ph}, 4-\mathrm{FC}_{6} \mathrm{H}_{4}, 4-\mathrm{BrC}_{6} \mathrm{H}_{4}, 4-\mathrm{MeOC}_{6} \mathrm{H}_{4}$, 2-furyl, 4- $\mathrm{MeC}_{6} \mathrm{H}_{4}$, 3,4,5-(OMe) $)_{3} \mathrm{C}_{6} \mathrm{H}_{2}, 4-\mathrm{N}(\mathrm{Me})_{2} \mathrm{C}_{6} \mathrm{H}_{4}$, 2-thienyl, 3- $\mathrm{NO}_{2} \mathrm{C}_{6} \mathrm{H}_{4}$, $2-\mathrm{NO}_{2} \mathrm{C}_{6} \mathrm{H}_{4}, 2-\mathrm{FC}_{6} \mathrm{H}_{4}$<smiles>[R]C1C(C#N)=C(N)Oc2cc(O)ccc21</smiles>
$\mathrm{R}=\mathrm{Ph}, 4-\mathrm{FC}_{6} \mathrm{H}_{4}, 4-\mathrm{BrC}_{6} \mathrm{H}_{4}, 4-\mathrm{MeOC}_{6} \mathrm{H}_{4}$, 2-furyl, 4- $\mathrm{MeC}_{6} \mathrm{H}_{4}, 3,4,5-(\mathrm{OMe})_{3} \mathrm{C}_{6} \mathrm{H}_{2}$, $4-\mathrm{N}(\mathrm{Me})_{2} \mathrm{C}_{6} \mathrm{H}_{4}$

\section{Scheme 1}




\section{Results and Discussion}

Although different synthetic strategies have been used for the synthesis of 2-amino- $4 H$ chromenes employing some bases, however, these methods suffer from the drawbacks such as low product yield, cumbersome isolation of the product and long reaction time. In order to develop a viable alternative, a variety of catalysts were first investigated for the typical multicomponent reaction of benzaldehyde $\mathbf{3 a}$, malononitrile and resorcinol under conventional as well as microwave irradiation conditions. The outcome is given in Table 1. The data reveals that DBU under conventional conditions brings about the reaction to afford the product $4 \mathrm{a}$ in $76 \%$ yield (Table 1, entry 14). The use of KF/Alumina also promoted the reaction to a reasonable extent $(62 \%$, Table 1 , entry 12$)$, but the other catalysts such as PTSA, TBAB, $\mathrm{NaHCO}_{3}$, guanidine did not work well (Table 1, entries 4, 6, 8 and 10). It is interesting to note that the afore mentioned reaction in the presence of DBU, when carried out in ethanol at room temperature, gave rise to $45 \%$ yield of the product 4a in 60 minutes. At $50{ }^{\circ} \mathrm{C}$, however, the conversion was found to be the optimum; a further increase in temperature could not improve the yield further. Application of monomode MW irradiation at the same temperature, however, brought about a commendable increase in the yield as well as a dramatic reduction in the reaction time, the best result being obtained using $100 \mathrm{~W}$ at $50^{\circ} \mathrm{C}$ in just 3 minutes in the presence of $5 \mathrm{~mol} \% \mathrm{DBU}$ ( $c f$. entry 14). A further increase in the MW power and temperature starts diminishing the yield (entries 15-16).

Under the optimized set of MW reaction conditions $\left(100 \mathrm{~W}\right.$ and $\left.50{ }^{\circ} \mathrm{C}\right)$, a number of aromatic aldehydes 3a-l were allowed to undergo multicomponent reaction with malononitrile and resorcinol in a molar ratio of 1:1:1 with DBU (5 mol\%) in ethanol affording 2-amino- $4 H$ chromenes 4a-l in excellent yields in 2 to 4 minutes (Table 2). The physical and spectral data of all the products are in full agreement with the assigned structures.

In another endeavor, we optimized the reaction of $\mathbf{4 a}$ with cyclohexanone 5 using various Lewis acid catalysts in different solvents (methanol, ethanol, acetonitrile, and dichloromethane) at reflux and under microwave conditions (Scheme 1 and Table 3). When the reaction was carried out using anhydrous $\mathrm{AlCl}_{3}$ (1.2 equiv) as a catalyst in $\mathrm{CH}_{2} \mathrm{Cl}_{2}$ under reflux as well as under MW, the desired pyranopyridine 6a was formed in 58\% under conventional heating (reflux) in 1.0 hour and $91 \%$ under $\mathrm{MW}$ at $45{ }^{\circ} \mathrm{C}$ in $8 \mathrm{~min}$. (Table 3, entry 1). Decreasing the molar proportion of $\mathrm{AlCl}_{3}$ decreases the yield of the product 6a considerably (Table 3, entries 23 ), whereas an increased equivalent of $\mathrm{AlCl}_{3}$ did not improve the yield further (Table 3, entry 4). Switching to other Lewis acids such as $\mathrm{FeCl}_{3}, \mathrm{ZnCl}_{2}, \mathrm{Yb}(\mathrm{OTf})_{3}, \mathrm{Sc}(\mathrm{OTf})_{3}, \mathrm{InCl}_{3}, \mathrm{I}_{2}$ and Montmorillonite K10 (Table 3, entries 8-14) and solvents such as ethanol, methanol and acetonitrile gave rise to no measurable products (Table 3, entries 5-7). It is concluded from the table that $\mathrm{AlCl}_{3}$ in $\mathrm{CH}_{2} \mathrm{Cl}_{2}$ under microwave irradiation $\left(150 \mathrm{~W}, 45^{\circ} \mathrm{C}\right)$ affords the best result with considerable reduction in the reaction time. Further increase of MW power as well as temperature did not improve the product yield. 
Various substituted chromenes 4 were made to react with cyclohexanone 5 using a molar ratio of 1:1.3 with $\mathrm{AlCl}_{3}$ (1.2 equivalent) in dry $\mathrm{CH}_{2} \mathrm{Cl}_{2}$ using safe pressure regulation 10-mL pressurized vials with "snap-on" cap under microwave $\left(150 \mathrm{~W}, 45^{\circ} \mathrm{C}\right)$ heating for $6-8$ minutes. The results are given in Table 4 . The progress of the reaction was monitored by TLC. After completion of reaction, the mixture was poured into a beaker containing ice cold water and was extracted with $\mathrm{CH}_{2} \mathrm{Cl}_{2}(3 \times 15 \mathrm{~mL})$. The organic phase was dried over anhydrous $\mathrm{Na}_{2} \mathrm{SO}_{4}$, filtered and concentrated under reduced pressure. The crude product was purified through column chromatography ( $40 \%$ ethyl acetate in hexane) to afford pure product $\mathbf{6}$ in good yield. It is important to mention that under the investigated set of reaction conditions, pyranopyridines 6 are exclusively obtained as the only isolable product with no measurable side product.

Table 1. Optimization of reaction conditions for the multi-component synthesis of $\mathbf{4 a}$

\begin{tabular}{lllllllll}
\hline Entry & Catalyst & \multicolumn{3}{c}{ Microwave } & \multicolumn{3}{c}{ Conventional } \\
\hline & & $\begin{array}{c}\text { MW } \\
(\text { Watt })\end{array}$ & $\begin{array}{c}\text { Temp. } \\
\left({ }^{\circ} \mathrm{C}\right)\end{array}$ & $\begin{array}{c}\text { Time } \\
(\min .)\end{array}$ & $\begin{array}{c}\text { Yield } \\
(\%)\end{array}$ & $\begin{array}{c}\text { Temp. } \\
\left({ }^{\circ} \mathrm{C}\right)\end{array}$ & $\begin{array}{c}\text { Time } \\
(\mathrm{min} .)\end{array}$ & $\begin{array}{c}\text { Yield } \\
(\%)\end{array}$ \\
\hline $\mathbf{1}$ & - & 80 & 50 & 20 & 25 & $\mathrm{RT}$ & 120 & - \\
$\mathbf{2}$ & - & 100 & 50 & 10 & 42 & Reflux & 120 & 38 \\
$\mathbf{3}$ & $\mathrm{PTSA}$ & 80 & 50 & 10 & 20 & $\mathrm{RT}$ & 120 & Trace \\
$\mathbf{4}$ & $\mathrm{PTSA}$ & 100 & 50 & 10 & 32 & 50 & 90 & 12 \\
$\mathbf{5}$ & $\mathrm{TBAB}$ & 80 & 50 & 15 & 28 & $\mathrm{RT}$ & 120 & - \\
$\mathbf{6}$ & $\mathrm{TBAB}$ & 100 & 50 & 10 & 44 & 50 & 120 & 20 \\
$\mathbf{7}$ & $\mathrm{NaHCO}$ & 80 & 50 & 10 & 68 & $\mathrm{RT}$ & 120 & 25 \\
$\mathbf{8}$ & $\mathrm{NaHCO}$ & 100 & 50 & 15 & 79 & 50 & 90 & 56 \\
$\mathbf{9}$ & $\mathrm{Guanidine}$ & 80 & 50 & 15 & 35 & $\mathrm{RT}$ & 120 & - \\
$\mathbf{1 0}$ & $\mathrm{Guanidine}$ & 100 & 50 & 10 & 48 & 50 & 120 & 15 \\
$\mathbf{1 1}$ & $\mathrm{KF} / \mathrm{Al}_{2} \mathrm{O}_{3}$ & 80 & 50 & 15 & 68 & $\mathrm{RT}$ & 120 & 30 \\
$\mathbf{1 2}$ & $\mathrm{KF} / \mathrm{Al}_{2} \mathrm{O}_{3}$ & 100 & 50 & 10 & 75 & 50 & 120 & 62 \\
$\mathbf{1 3}$ & $\mathrm{DBU}$ & 80 & 50 & 10 & 85 & $\mathrm{RT}$ & 60 & 45 \\
$\mathbf{1 4}$ & $\mathrm{DBU}$ & 100 & 50 & 03 & 94 & 50 & 40 & 76 \\
$\mathbf{1 5}$ & $\mathrm{DBU}$ & 150 & 50 & 05 & 90 & Reflux & 30 & 78 \\
$\mathbf{1 6}$ & $\mathrm{DBU}$ & 150 & 80 & 05 & 87 & - & - & - \\
\hline
\end{tabular}

${ }^{a}$ Isolated mass yield based on resorcinol 
Table 2. Synthesis of 2-amino-4H-chromenes 4a-l

\begin{tabular}{|c|c|c|c|c|c|}
\hline \multirow[t]{2}{*}{ Product } & \multirow[t]{2}{*}{$\mathrm{R}$} & \multicolumn{2}{|c|}{ Microwave $\left(100 \mathrm{~W}, 50^{\circ} \mathrm{C}\right)$} & \multicolumn{2}{|c|}{$\mathrm{DBU}, 50^{\circ} \mathrm{C}$} \\
\hline & & Time (min.) & Yield $(\%)^{\mathrm{a}}$ & Time(min.) & Yield (\%) ${ }^{\mathrm{a}}$ \\
\hline $4 a$ & $\mathrm{Ph}$ & 3 & 94 & 40 & 76 \\
\hline $4 \mathrm{~b}$ & $4-\mathrm{FC}_{6} \mathrm{H}_{4}$ & 2 & 89 & 35 & 62 \\
\hline $4 c$ & $4-\mathrm{BrC}_{6} \mathrm{H}_{4}$ & 3 & 93 & 35 & 74 \\
\hline $4 d$ & $4-\mathrm{MeOC}_{6} \mathrm{H}_{4}$ & 3 & 90 & 35 & 60 \\
\hline $4 e$ & 2-furyl & 2 & 92 & 45 & 63 \\
\hline $4 f$ & 4- $-\mathrm{MeC}_{6} \mathrm{H}_{4}$ & 4 & 91 & 50 & 65 \\
\hline $4 g$ & $3,4,5-(\mathrm{MeO})_{3} \mathrm{C}_{6} \mathrm{H}_{2}$ & 4 & 90 & 45 & 60 \\
\hline $4 h$ & $4-\mathrm{N}(\mathrm{Me})_{2} \mathrm{C}_{6} \mathrm{H}_{4}$ & 4 & 88 & 45 & 62 \\
\hline $4 i$ & 2-thienyl & 3 & 96 & 45 & 70 \\
\hline $4 j$ & $3-\mathrm{NO}_{2} \mathrm{C}_{6} \mathrm{H}_{4}$ & 3 & 87 & 40 & 72 \\
\hline $4 \mathbf{k}$ & $2-\mathrm{NO}_{2} \mathrm{C}_{6} \mathrm{H}_{4}$ & 4 & 89 & 30 & 62 \\
\hline 41 & $2-\mathrm{FC}_{6} \mathrm{H}_{4}$ & 3 & 87 & 35 & 72 \\
\hline
\end{tabular}

${ }^{\mathrm{a}}$ Isolated mass yield based on resorcinol

Table 3. Optimization of the reaction conditions using compound 4a as reference

\begin{tabular}{|c|c|c|c|c|c|}
\hline \multirow[t]{3}{*}{ Entry } & \multirow[t]{3}{*}{ Lewis Acid } & \multicolumn{4}{|c|}{ Reaction Condition } \\
\hline & & \multicolumn{2}{|c|}{ Reflux } & \multicolumn{2}{|c|}{$\mathrm{MW}^{\mathrm{a}}$} \\
\hline & & Time (h) & Yield (\%) & Time (min) & Yield $(\%)$ \\
\hline 1 & $\mathrm{AlCl}_{3}$ (1.2 equiv) & 2.0 & 58 & 8 & 91 \\
\hline 2 & $\mathrm{AlCl}_{3}(0.5$ equiv $)$ & 2.0 & 15 & 10 & 25 \\
\hline 3 & $\mathrm{AlCl}_{3}$ (1.0 equiv) & 2.0 & 45 & 10 & 78 \\
\hline 4 & $\mathrm{AlCl}_{3}$ (1.5 equiv) & 2.0 & 57 & 8 & 91 \\
\hline 5 & $\mathrm{AlCl}_{3}(1.2 \text { equiv })^{\mathrm{b}}$ & 2.5 & - & 10 & Trace \\
\hline 6 & $\mathrm{AlCl}_{3}(1.2 \text { equiv })^{\mathrm{c}}$ & 2.5 & - & 10 & Trace \\
\hline 7 & $\mathrm{AlCl}_{3}(1.2 \text { equiv })^{\mathrm{d}}$ & 2.5 & - & 10 & - \\
\hline 8 & $\mathrm{FeCl}_{3}(1.5$ equiv $)$ & 1.5 & - & 8 & - \\
\hline 9 & $\mathrm{ZnCl}_{2}(1.5$ equiv $)$ & 2.0 & - & 8 & - \\
\hline 10 & $\mathrm{Sc}(\mathrm{OTf})_{3}$ & 2.5 & - & 10 & - \\
\hline 11 & $\mathrm{Yb}(\mathrm{OTf})_{3}$ & 2.0 & - & 8 & - \\
\hline 12 & $\mathrm{InCl}_{3}$ & 2.0 & - & 8 & - \\
\hline 13 & $\mathrm{I}_{2}$ & 2.5 & - & 10 & - \\
\hline 14 & MontmorilloniteK10 & 2.0 & - & 10 & - \\
\hline
\end{tabular}

${ }^{\mathrm{a}} \mathrm{MW}$ heating performed on $150 \mathrm{Watt}$ power and $45^{\circ} \mathrm{C}$ temperature

${ }^{\mathrm{b}}$ Reaction was carried out in ethanol

${ }^{\mathrm{c}}$ Reaction was carried out in methanol

${ }^{\mathrm{d}}$ Reaction was carried out in acetonitrile 
Table 4. Microwave assisted synthesis ${ }^{a}$ of pyrano[2,3-b]pyridine 6a-h

\begin{tabular}{|c|c|c|c|c|c|}
\hline Entry & Reactant 4 & Product 6 & Time (min.) & Yield $(\%)^{b}$ & $\mathrm{Mp}\left({ }^{\circ} \mathrm{C}\right)$ \\
\hline $\mathrm{a}$ & & & 8 & 91 & $313-315$ \\
\hline b & & & 7 & 89 & 292-294 \\
\hline $\mathrm{c}$ & & & 8 & 93 & $281-282$ \\
\hline d & & & 6 & 92 & $295-297$ \\
\hline e & & & 8 & 89 & $318-320$ \\
\hline $\mathrm{f}$ & & & 7 & 90 & $282-284$ \\
\hline g & & & 8 & 87 & $285-287$ \\
\hline $\mathrm{h}$ & & & 8 & 85 & $>320$ \\
\hline
\end{tabular}

${ }^{a}$ Microwave heating performed on $150 \mathrm{Watt}$ power and $45^{\circ} \mathrm{C}$ temperature

${ }^{\mathrm{b}}$ Isolated mass yield based on chromenes 4 
In conclusion, the present report demonstrates a facile DBU-catalysed multicomponent condensation of resorcinol, malononitrile and aromatic aldehydes to afford 2-amino-4H-3-cyanochromenes, which are subsequently made to react with cyclohexanone to furnish novel pyranopyridine derivatives in the presence of aluminium chloride. The advantages include improved yield, easier work-up, shorter reaction time and mild reaction conditions.

\section{Experimental Section}

General. IR spectra were recorded on a JASCO FT/IR-5300 spectrophotometer, whereas NMR was run on a JEOL AL300 FTNMR spectrometer. The chemical shifts are given in $\delta$ ppm with respect to TMS as internal standard. Elemental analysis $(\mathrm{C}, \mathrm{H}, \mathrm{N})$ for final compounds were performed on a model CE-440 CHN analyzer. The tlc spots were detected using iodine chamber. Microwave irradiation was made using a CEM Discover single mode microwave reactor (Benchmate Model, USA) with infrared temperature probe and adjustable 0-300 W output power. All commercially available chemicals were purchased from Aldrich and Merck.

\section{General microwave procedure for the preparation of 2-amino-3-cyano-4H-chromenes 4a-I}

An equimolar mixture of resorcinol 1 ( $1 \mathrm{mmol})$, malononitrile 2 (1 mmol) and aromatic aldehyde 3a-l ( $1 \mathrm{mmol})$ was placed in a sealed pressure regulation $10-\mathrm{mL}$ pressurized vial containing ethanol (2 mL) and DBU (5 mol\%) with "snap-on" cap and was irradiated in the single-mode microwave synthesis system at $100 \mathrm{~W}$ power and $50{ }^{\circ} \mathrm{C}$ temperature for $2-4$ minutes. After completion of the reaction (TLC), the mixture was poured into ice cold water. The resulting precipitate was filtered, dried and recrystallized from ethanol to afford pure 2-amino- $4 \mathrm{H}$ chromenes 4a-l.

2-Amino-3-cyano-7-hydroxy-4-phenyl-4H-chromene (4a). Light yellow solid, mp: 231$233{ }^{\circ} \mathrm{C}$ (Lit. ${ }^{26} \mathrm{mp}: 232-234{ }^{\circ} \mathrm{C}$ ). IR (KBr): 3427, 3218, 2190, 1650, $1580 \mathrm{~cm}^{-1}$. ${ }^{1} \mathrm{H}$ NMR $(300$ MHz, DMSO-d $\left.)_{6}\right): \delta=4.61(\mathrm{~s}, 1 \mathrm{H}, \mathrm{H}-4), 6.85\left(\mathrm{~s}, 2 \mathrm{H}, \mathrm{NH}_{2}\right), 6.40-7.31(\mathrm{~m}, 8 \mathrm{H}, \mathrm{ArH}), 9.68(\mathrm{~s}, 1 \mathrm{H}$, $\mathrm{OH}) .{ }^{13} \mathrm{C}$ NMR $\left(75 \mathrm{MHz}, \mathrm{DMSO}-d_{6}\right): \delta=56.2,102.1,112.3,113.7,120.6,126.6,127.3,128.5$, 129.8, 146.3, 148.8, 157.0, 160.2. Anal. Calcd for $\mathrm{C}_{16} \mathrm{H}_{12} \mathrm{~N}_{2} \mathrm{O}_{2}: \mathrm{C}, 72.72 ; \mathrm{H}, 4.58 ; \mathrm{N}, 10.60$. Found: C, 72.45; H, 4.38; N, 10.49 .

2-Amino-3-cyano-7-hydroxy-4-(4-flurophenyl)-4H-chromene (4b). Yellow solid, mp: 186$188{ }^{\circ} \mathrm{C}$ (Lit. ${ }^{27} \mathrm{mp}: 187-189{ }^{\circ} \mathrm{C}$ ). IR (KBr): 3430, 3342, 2187, 1645, $1587 \mathrm{~cm}^{-1}{ }^{1} \mathrm{H}$ NMR $(300$ MHz, DMSO-d $)_{6}: \delta=4.65$ (s, 1H, H-4), 6.85 (s, 2H, NH $), 6.40-7.21(\mathrm{~m}, 7 \mathrm{H}, \mathrm{ArH}), 9.71(\mathrm{~s}, 1 \mathrm{H}$, $\mathrm{OH}) .{ }^{13} \mathrm{C}$ NMR $\left(75 \mathrm{MHz}, \mathrm{DMSO}-d_{6}\right): \delta=56.6,102.6,112.8,113.9,121.0,129.6,129.7,130.3$, 143.0, 148.7, 157.3, 159.8, 160.6. Anal. Calcd for $\mathrm{C}_{16} \mathrm{H}_{11} \mathrm{FN}_{2} \mathrm{O}_{2}$ : C, 68.08; H, 3.93; N, 9.92. Found: C, 67.94; H, 3.84; N, 10.12 .

2-Amino-3-cyano-7-hydroxy-4-(4-bromophenyl)-4H-chromene (4c). Light yellow solid, mp: 224-226 ${ }^{\circ} \mathrm{C}$ (Lit. ${ }^{27} \mathrm{mp}: 225-227{ }^{\circ} \mathrm{C}$ ). IR (KBr): 3471, 3336, 2194, 1641, $1578 \mathrm{~cm}^{-1}$. ${ }^{1} \mathrm{H}$ NMR (300 MHz, DMSO-d $\left.)_{6}\right): \delta=4.64(\mathrm{~s}, 1 \mathrm{H}, \mathrm{H}-4), 6.90\left(\mathrm{~s}, 2 \mathrm{H}, \mathrm{NH}_{2}\right), 6.40-7.84(\mathrm{~m}, 7 \mathrm{H}, \mathrm{ArH}), 9.71$ (s, 
$1 \mathrm{H}, \mathrm{OH}) .{ }^{13} \mathrm{C}$ NMR $\left(75 \mathrm{MHz}, \mathrm{DMSO}-d_{6}\right): \delta=56.4,102.6,112.0,113.7,120.2,121.4,130.1$, 130.3, 132.0, 147.2, 149.5, 157.8, 160.5. Anal. Calcd for $\mathrm{C}_{16} \mathrm{H}_{11} \mathrm{BrN}_{2} \mathrm{O}_{2}: \mathrm{C}, 56.0 ; \mathrm{H}, 3.23 ; \mathrm{N}$, 8.16. Found: C, 55.72; H, 3.14; N, 8.05.

2-Amino-3-cyano-7-hydroxy-4-(4-methoxyphenyl)-4H-chromene (4d). Yellow solid, mp: 110-112 ${ }^{\circ} \mathrm{C}$ (Lit. ${ }^{34} \mathrm{mp}: 112-114{ }^{\circ} \mathrm{C}$ ). IR (KBr): 3424, 3333, 2190, 1651, $1587 \mathrm{~cm}^{-1} .{ }^{1} \mathrm{H}$ NMR (300 MHz, DMSO- $\left.d_{6}\right): \delta=3.51\left(\mathrm{~s}, 3 \mathrm{H}, \mathrm{OCH}_{3}\right), 4.53(\mathrm{~s}, 1 \mathrm{H}, \mathrm{H}-4), 6.84\left(\mathrm{~s}, 2 \mathrm{H}, \mathrm{NH}_{2}\right), 6.43-7.21$ $(\mathrm{m}, 7 \mathrm{H}, \mathrm{ArH}), 9.63(\mathrm{~s}, 1 \mathrm{H}, \mathrm{OH}) .{ }^{13} \mathrm{C}$ NMR $\left(75 \mathrm{MHz}, \mathrm{DMSO}-d_{6}\right): \delta=55.9,56.0,102.2,112.3$, $113.5,120.6,126.4,129.7,136.5,142.0,148.4,152.7,156.8,160.4$. Anal. Calcd for $\mathrm{C}_{17} \mathrm{H}_{14} \mathrm{~N}_{2} \mathrm{O}_{3}: \mathrm{C}, 69.38 ; \mathrm{H}, 4.79 ; \mathrm{N}, 9.52$. Found: $\mathrm{C}, 69.16 ; \mathrm{H}, 4.54 ; \mathrm{N}, 9.48$.

2-Amino-3-cyano-7-hydroxy-4-(2-furyl)-4H-chromene (4e). Yellow solid, mp: $189-191{ }^{\circ} \mathrm{C}$. IR (KBr): $3420,3331,2193,1651,1589 \mathrm{~cm}^{-1} .{ }^{1} \mathrm{H}$ NMR $\left(300 \mathrm{MHz}\right.$, DMSO- $\left.d_{6}\right): \delta=4.75(\mathrm{~s}, 1 \mathrm{H}$, $\mathrm{H}-4), 6.92$ (s, $\left.2 \mathrm{H}, \mathrm{NH}_{2}\right), 6.13-7.50(\mathrm{~m}, 6 \mathrm{H}, \mathrm{ArH}), 9.74(\mathrm{~s}, 1 \mathrm{H}, \mathrm{OH}) .{ }^{13} \mathrm{C}$ NMR $(75 \mathrm{MHz}$, DMSO$\left.d_{6}\right): \delta=53.2,102.3,105.4,110.2,111.0,112.2,120.3,129.5,142.3,149.1,156.9,157.3,160.9$. Anal. Calcd for $\mathrm{C}_{14} \mathrm{H}_{10} \mathrm{~N}_{2} \mathrm{O}_{3}$ : C, 66.14; H, 3.96; N, 11.02. Found: C, 65.93; H, 3.84; N, 10.87 .

2-Amino-3-cyano-7-hydroxy-4-(4-methylphenyl)-4H-chromene (4f). Yellow solid, mp: 184$186{ }^{\circ} \mathrm{C}$. IR (KBr): $3409,3332,2194,1656,1589 \mathrm{~cm}^{-1} .{ }^{1} \mathrm{H}$ NMR (300 MHz, DMSO-d $\left.d_{6}\right): \delta=2.24$ (s, $\left.3 \mathrm{H}, \mathrm{CH}_{3}\right), 4.56(\mathrm{~s}, 1 \mathrm{H}, \mathrm{H}-4), 6.81\left(\mathrm{~s}, 2 \mathrm{H}, \mathrm{NH}_{2}\right), 6.38-7.10(\mathrm{~m}, 7 \mathrm{H}, \mathrm{ArH}), 9.66(\mathrm{~s}, 1 \mathrm{H}, \mathrm{OH}) .{ }^{13} \mathrm{C}$ NMR $\left(75 \mathrm{MHz}, \mathrm{DMSO}-\mathrm{d}_{6}\right): \delta=20.5,56.4,102.1,112.3,113.8,120.6,127.2,129.5,129.8$, 135.6, 143.4, 148.7, 156.9, 160.1. Anal. Calcd for $\mathrm{C}_{17} \mathrm{H}_{14} \mathrm{~N}_{2} \mathrm{O}_{2}: \mathrm{C}, 73.37 ; \mathrm{H}, 5.07 ; \mathrm{N}, 10.07$. Found: C, 73.12; H, 4.85 N, 9.86 .

2-Amino-3-cyano-7-hydroxy-4-(3,4,5-trimethoxyphenyl)-4H-chromene (4g). Yellow solid, mp: $208-210^{\circ} \mathrm{C}$. IR (KBr): 3473, 3335, 2199, 1647, $1589 \mathrm{~cm}^{-1} .{ }^{1} \mathrm{H}$ NMR (300 MHz, DMSO-d $\left.{ }_{6}\right)$ : $\delta=3.62\left(\mathrm{~s}, 3 \mathrm{H}, \mathrm{OCH}_{3}\right), 3.71\left(\mathrm{~s}, 6 \mathrm{H}, \mathrm{OCH}_{3}\right), 4.57(\mathrm{~s}, 1 \mathrm{H}, \mathrm{H}-4), 6.86\left(\mathrm{~s}, 2 \mathrm{H}, \mathrm{NH}_{2}\right), 6.38-6.89(\mathrm{~m}$, $5 \mathrm{H}, \mathrm{ArH}), 9.68(\mathrm{~s}, 1 \mathrm{H}, \mathrm{OH}) .{ }^{13} \mathrm{C}$ NMR $\left(75 \mathrm{MHz}, \mathrm{DMSO}-d_{6}\right): \delta=55.8,56.0,59.9,102.2,104.6$, $112.3,113.5,120.6,129.8,136.2,141.9,148.6,152.9,157.0,160.3$. Anal. Calcd for $\mathrm{C}_{19} \mathrm{H}_{18} \mathrm{~N}_{2} \mathrm{O}_{5}: \mathrm{C}, 64.40 ; \mathrm{H}, 5.12 ; \mathrm{N}, 7.91$. Found: $\mathrm{C}, 64.15 ; \mathrm{H}, 4.92 . \mathrm{N}, 7.86$.

2-Amino-3-cyano-7-hydroxy-4-(4-dimethylaminophenyl)-4H-chromene (4h). Yellow solid, mp: 193-195 ${ }^{\circ} \mathrm{C}$. IR (KBr): 3415, 3330, 2190, 1654, $1585 \mathrm{~cm}^{-1} .{ }^{1} \mathrm{H}$ NMR (300 MHz, DMSO$\left.d_{6}\right): \delta=2.84\left(\mathrm{~s}, 6 \mathrm{H}, \mathrm{N}\left(\mathrm{CH}_{3}\right)_{2}\right), 4.46(\mathrm{~s}, 1 \mathrm{H}, \mathrm{H}-4), 6.77$ (s, $\left.2 \mathrm{H}, \mathrm{NH}_{2}\right), 6.37-6.97(\mathrm{~m}, 7 \mathrm{H}, \mathrm{ArH})$, $9.66(\mathrm{~s}, 1 \mathrm{H}, \mathrm{OH}) .{ }^{13} \mathrm{C}$ NMR $\left(75 \mathrm{MHz}, \mathrm{DMSO}-d_{6}\right): \delta=40.2,56.8,101.9,112.1,112.5,114.4$, 120.7, 127.8, 129.8, 134.1, 148.7, 149.2, 156.7, 159.9. Anal. Calcd for $\mathrm{C}_{18} \mathrm{H}_{17} \mathrm{~N}_{3} \mathrm{O}_{2}: \mathrm{C}, 70.34 ; \mathrm{H}$, 5.58; N, 13.67. Found: C, 70.15; H, 5.36; N, 13.51 .

2-Amino-3-cyano-7-hydroxy-4-(2-thienyl)-4H-chromene (4i). Yellow solid, mp: $204-205{ }^{\circ} \mathrm{C}$ (Lit. ${ }^{34} \mathrm{mp}: 202-204{ }^{\circ} \mathrm{C}$ ). IR (KBr): 3422, 3332, 2193, 1653, $1568 \mathrm{~cm}^{-1} .{ }^{1} \mathrm{H}$ NMR $(300 \mathrm{MHz}$, DMSO-d $\left.{ }_{6}\right): \delta=4.97(\mathrm{~s}, 1 \mathrm{H}, \mathrm{H}-4), 6.91\left(\mathrm{~s}, 2 \mathrm{H}, \mathrm{NH}_{2}\right), 6.38-7.35(\mathrm{~m}, 6 \mathrm{H}, \mathrm{ArH}), 9.76(\mathrm{~s}, 1 \mathrm{H}, \mathrm{OH})$. ${ }^{13} \mathrm{C}$ NMR $\left(75 \mathrm{MHz}\right.$, DMSO- $\left.d_{6}\right): \delta=56.4,102.2,112.4,113.4,120.5,124.0,125.0,126.7,129.8$, 148.5, 151.5, 157.4, 160.3. Anal. Calcd for $\mathrm{C}_{14} \mathrm{H}_{10} \mathrm{~N}_{2} \mathrm{O}_{2} \mathrm{~S}: \mathrm{C}, 62.21 ; \mathrm{H}, 3.73 ; \mathrm{N}, 10.36$. Found: $\mathrm{C}$, $62.28 ; \mathrm{H}, 3.70 ; \mathrm{N}, 10.25$. 
2-Amino-3-cyano-7-hydroxy-4-(3-nitrophenyl)-4H-chromene (4j). Yellow solid, mp: 169$170{ }^{\circ} \mathrm{C}$. IR (KBr): 3440, 3345, 2192, 1650, $1585 \mathrm{~cm}^{-1} .{ }^{1} \mathrm{H}$ NMR $\left(300 \mathrm{MHz}, \mathrm{DMSO}-d_{6}\right): \delta=4.85$ (s, 1H, H-4), 7.05 (s, 2H, NH $), 6.45-8.10(\mathrm{~m}, 7 \mathrm{H}, \mathrm{ArH}), 9.78(\mathrm{~s}, 1 \mathrm{H}, \mathrm{OH}) .{ }^{13} \mathrm{C} \mathrm{NMR}(75 \mathrm{MHz}$, DMSO- $\left.d_{6}\right): \delta=55.2,102.4,111.9$ 112.7, 120.0, 121.0, 123.7, 128.2, 129.6, 131.4, 133.4, 139.3, 148.7, 149.0, 157.5, 160.3. Anal. Calcd. for $\mathrm{C}_{16} \mathrm{H}_{11} \mathrm{~N}_{3} \mathrm{O}_{4}$ : C, 62.14; H, 3.58; N, 13.59. Found: C, $61.87 ; \mathrm{H}, 3.67 ; \mathrm{N}, 13.25$.

2-Amino-3-cyano-7-hydroxy-4-(2-nitrophenyl)-4H-chromene (4k). Yellow solid, mp: 162$163{ }^{\circ} \mathrm{C}$. IR (KBr): 3417, 3342, 2187, 1650, $1590 \mathrm{~cm}^{-1} .{ }^{1} \mathrm{H}$ NMR $(300 \mathrm{MHz}$, DMSO-d $): \delta=5.14$ (s, 1H, H-4), 7.0 (s, 2H, NH $), 6.43-7.86(\mathrm{~m}, 7 \mathrm{H}, \mathrm{ArH}), 9.80(\mathrm{~s}, 1 \mathrm{H}, \mathrm{OH}) .{ }^{13} \mathrm{C} \mathrm{NMR}(75 \mathrm{MHz}$, DMSO- $\left.d_{6}\right): \delta=55.5,102.7,112.1,113.0,120.4,121.2,123.5,128.0,129.5,131.7,133.2,139.5$, 148.3, 149.2, 157.8, 160.4. Anal. Calcd for $\mathrm{C}_{16} \mathrm{H}_{11} \mathrm{~N}_{3} \mathrm{O}_{4}$ : C, 62.14; H, 3.58; N, 13.59. Found: C, $61.93 ; \mathrm{H}, 3.50 ; \mathrm{N}, 13.42$.

2-Amino-3-cyano-7-hydroxy-4-(2-fluorophenyl)-4H-chromene (4l). Yellow solid, mp: 200$202{ }^{\circ} \mathrm{C}$. IR (KBr): 3424, 3333, 2190, 1651, $1587 \mathrm{~cm}^{-1} .{ }^{1} \mathrm{H}$ NMR (300 MHz, DMSO- $\left.d_{6}\right): \delta=4.87$ $(\mathrm{s}, 1 \mathrm{H}, \mathrm{H}-4), 6.86\left(\mathrm{~s}, 2 \mathrm{H}, \mathrm{NH}_{2}\right), 6.38-7.25(\mathrm{~m}, 7 \mathrm{H}, \mathrm{ArH}), 9.69(\mathrm{~s}, 1 \mathrm{H}, \mathrm{OH}) .{ }^{13} \mathrm{C} \mathrm{NMR}(75 \mathrm{MHz}$, DMSO- $\left.d_{6}\right): \delta=54.5,102.2,112.4,115.5,115.8,120.4,124.7,128.8,129.5,129.8,132.7,149.1$, 157.2, 160.6. Anal. Calcd for $\mathrm{C}_{16} \mathrm{H}_{11} \mathrm{FN}_{2} \mathrm{O}_{2}$ : C, 68.08; H, 3.93; N, 9.92. Found: C, 67.73; H, $3.80 ; \mathrm{N}, 9.75$.

General microwave procedure for the synthesis of substituted tetrahydro-8H-5-oxa-6-azanaphthacen-3-ols 6a-h

A mixture of substituted 2-amino-3-cyano-4H-chromene 4 (1 mmol), cyclohexanone 5 $(1.3 \mathrm{mmol})$ and anhydrous $\mathrm{AlCl}_{3}(1.2 \mathrm{mmol})$ was placed in a sealed pressure regulation $10 \mathrm{~mL}$ pressurized vial containing dry dichloromethane $(2 \mathrm{~mL})$ with "snap-on" cap and was irradiated in the single-mode microwave synthesis system using $150 \mathrm{~W}$ power at $45^{\circ} \mathrm{C}$ temperature for $6-8$ minutes. After completion of reaction (TLC), the reaction mixture was poured into a beaker containing ice cold water. The mixture was extracted with $\mathrm{CH}_{2} \mathrm{Cl}_{2}(3 \times 15 \mathrm{~mL})$, dried over anhydrous $\mathrm{Na}_{2} \mathrm{SO}_{4}$ and filtered. The solvent was evaporated under reduced pressure and the resultant crude product was purified by column chromatography ( $40 \%$ ethyl acetate in hexane) to afford pure product 6.

11-Amino-12-phenyl-7,9,10,12-tetrahydro-8H-5-oxa-6-aza-naphthacen-3-ol (6a). White solid, mp: $313-315{ }^{\circ} \mathrm{C}$. IR (KBr): 3456, 3380, 2928, 1624, 1462, 1238, $1172 \mathrm{~cm}^{-1}$. ${ }^{1} \mathrm{H}$ NMR (300 MHz, DMSO-d $\left.)_{6}\right): \delta=1.70\left(\mathrm{~m}, 4 \mathrm{H}, \mathrm{CH}_{2}\right), 2.22\left(\mathrm{~m}, 2 \mathrm{H}, \mathrm{CH}_{2}\right), 2.50\left(\mathrm{~m}, 2 \mathrm{H}, \mathrm{CH}_{2}\right), 5.20(\mathrm{~s}, 1 \mathrm{H}$, $\mathrm{CH}), 5.49$ (s, 2H, NH 2$), 6.42-7.22(\mathrm{~m}, 8 \mathrm{H}, \mathrm{ArH}), 9.52(\mathrm{~s}, 1 \mathrm{H}, \mathrm{OH}) .{ }^{13} \mathrm{C}$ NMR $(75 \mathrm{MHz}, \mathrm{DMSO}-$ $\left.d_{6}\right): \delta=22.2,22.4,22.9,32.0,38.2,98.6,102.6,111.1,112.0,116.0,126.3,127.1,128.5,129.5$, 146.0, 151.2, 151.3, 152.3, 155.4, 156.9. Anal. Calcd for $\mathrm{C}_{22} \mathrm{H}_{20} \mathrm{~N}_{2} \mathrm{O}_{2}$ : C, 76.72; H, 5.85; N, 8.13. Found: C, 76.58; H, 5.72; N, 7.92.

11-Amino-12-(4-fluorophenyl)-7,9,10,12-tetrahydro-8H-5-oxa-6-aza-naphthacen-3-ol (6b). White solid, mp: $292-294{ }^{\circ} \mathrm{C}$. IR (KBr): 3485, 3395, 2924, 1625, 1465, 1235, $1168 \mathrm{~cm}^{-1} .{ }^{1} \mathrm{H}$ NMR (300 MHz, DMSO-d $\left.)_{6}\right): \delta=1.70\left(\mathrm{~m}, 4 \mathrm{H}, \mathrm{CH}_{2}\right), 2.10\left(\mathrm{~m}, 2 \mathrm{H}, \mathrm{CH}_{2}\right), 2.53\left(\mathrm{~m}, 2 \mathrm{H}, \mathrm{CH}_{2}\right)$, 
$5.23(\mathrm{~s}, 1 \mathrm{H}, \mathrm{CH}), 5.53\left(\mathrm{~s}, 2 \mathrm{H}, \mathrm{NH}_{2}\right), 6.44-7.27(\mathrm{~m}, 7 \mathrm{H}, \mathrm{ArH}), 9.65(\mathrm{~s}, 1 \mathrm{H}, \mathrm{OH}) .{ }^{13} \mathrm{C}$ NMR $(75$ MHz, DMSO- $\left.d_{6}\right): \delta=22.1,22.3,22.9,32.0,37.7,98.2,102.7,111.3,112.1,115.2,119.3,127.3$, 129.3, 131.5, 145.6, 151.2, 151.3, 152.6, 155.5, 157.3. Anal. Calcd for $\mathrm{C}_{22} \mathrm{H}_{19} \mathrm{FN}_{2} \mathrm{O}_{2}: \mathrm{C}, 72.91$; H, 5.28; N, 7.73. Found: C, 72.83; H, 5.15; N, 7.58.

11-Amino-12-(4-bromophenyl)-7,9,10,12-tetrahydro-8H-5-oxa-6-aza-naphthacen-3-ol (6c). Yellow solid, mp: $281-282{ }^{\circ} \mathrm{C}$. IR (KBr): 3463, 3390, 2928, 1627, 1462, 1240, $1176 \mathrm{~cm}^{-1} .{ }^{1} \mathrm{H}$ NMR (300 MHz, DMSO- $\left.d_{6}\right): \delta=1.70\left(\mathrm{~m}, 4 \mathrm{H}, \mathrm{CH}_{2}\right), 2.22\left(\mathrm{~m}, 2 \mathrm{H}, \mathrm{CH}_{2}\right), 2.50\left(\mathrm{~m}, 2 \mathrm{H}, \mathrm{CH}_{2}\right), 5.24$ $(\mathrm{s}, 1 \mathrm{H}, \mathrm{CH}), 5.55\left(\mathrm{~s}, 2 \mathrm{H}, \mathrm{NH}_{2}\right), 6.43-7.41(\mathrm{~m}, 7 \mathrm{H}, \mathrm{ArH}), 9.69(\mathrm{~s}, 1 \mathrm{H}, \mathrm{OH}) .{ }^{13} \mathrm{C} \mathrm{NMR}(75 \mathrm{MHz}$, DMSO- $\left.d_{6}\right): \delta=22.1,22.3,22.9,32.0,37.8,98.2,102.7,111.3,112.0,115.2,119.3,127.3129 .3$, 131.32, 145.4, 151.2, 151.3, 152.4, 155.3, 157.2. Anal. Calcd for $\mathrm{C}_{22} \mathrm{H}_{19} \mathrm{BrN}_{2} \mathrm{O}_{2}$ : C, 62.42; $\mathrm{H}$, 4.52; N, 6.62. Found: C, 62.23; H, 4.61; N, 6.48.

11-Amino-12-(4-methoxyphenyl)-7,9,10,12-tetrahydro-8H-5-oxa-6-aza-naphthacen-3-ol (6d). White solid, mp: $295-29{ }^{\circ} \mathrm{C}$. IR (KBr): 3495, 3402, 2928, 1635, 1465, 1253, $1180 \mathrm{~cm}^{-1}$. ${ }^{1} \mathrm{H}$ NMR (300 MHz, DMSO-d $): \delta=1.68\left(\mathrm{~m}, 4 \mathrm{H}, \mathrm{CH}_{2}\right), 2.20\left(\mathrm{~m}, 2 \mathrm{H}, \mathrm{CH}_{2}\right), 2.54\left(\mathrm{~m}, 2 \mathrm{H}, \mathrm{CH}_{2}\right)$, 3.62(s, 3H, $\left.\mathrm{OCH}_{3}\right), 5.11(\mathrm{~s}, 1 \mathrm{H}, \mathrm{CH}), 5.44\left(\mathrm{~s}, 2 \mathrm{H}, \mathrm{NH}_{2}\right), 6.40-7.13(\mathrm{~m}, 7 \mathrm{H}, \mathrm{ArH}), 9.63(\mathrm{~s}, 1 \mathrm{H}$, $\mathrm{OH}) .{ }^{13} \mathrm{C}$ NMR $\left(75 \mathrm{MHz}, \mathrm{DMSO}-d_{6}\right): \delta=22.2,22.4,23.0,32.0,37.7,55.0,98.9,102.6,111.1$, $112.0,113.8,116.2,128.1,129.5,138.2,151.1,151.3,152.1,155.3,156.8,157.7$. Anal. Calcd for $\mathrm{C}_{23} \mathrm{H}_{22} \mathrm{~N}_{2} \mathrm{O}_{3}$ : C, 73.78; H, 5.92; N, 7.48. Found: C, 73.52; H, 5.80; N, 7.34.

11-Amino-12-furan-2-yl-7,9,10,12-tetrahydro-8H-5-oxa-6-aza-naphthacen-3-ol (6e). Brown solid, mp: $318-320{ }^{\circ} \mathrm{C}$. IR (KBr): 3491, 3402, 2935, 1627,1462, 1241, $1172 \mathrm{~cm}^{-1} .{ }^{1} \mathrm{H}$ NMR (300 MHz, DMSO-d $\left.d_{6}\right): \delta=1.69\left(\mathrm{~m}, 4 \mathrm{H}, \mathrm{CH}_{2}\right), 2.26\left(\mathrm{~m}, 2 \mathrm{H}, \mathrm{CH}_{2}\right), 2.48\left(\mathrm{~m}, 2 \mathrm{H}, \mathrm{CH}_{2}\right), 5.58(\mathrm{~s}, 1 \mathrm{H}$, $\mathrm{CH}), 5.68\left(\mathrm{~s}, 2 \mathrm{H}, \mathrm{NH}_{2}\right), 6.44-7.19(\mathrm{~m}, 6 \mathrm{H}, \mathrm{ArH}), 9.57$ (s, 1H, OH). ${ }^{13} \mathrm{C}$ NMR $(75 \mathrm{MHz}, \mathrm{DMSO}-$ $\left.d_{6}\right): \delta=22.5,22.8,23.4,32.4,34.2,99.0,103.1,111.5,112.3,116.0,124.2,124.8,126.7,129.7$, 150.7, 151.7, 151.9, 152.7, 157.6. Anal. Calcd for $\mathrm{C}_{20} \mathrm{H}_{18} \mathrm{~N}_{2} \mathrm{O}_{3}$ : C, 71.84; $\mathrm{H}, 5.43 ; \mathrm{N}, 8.38$. Found: C, 71.65; H, 5.38; N, 8.25.

11-Amino-12-(4-methylphenyl)-7,9,10,12-tetrahydro-8H-5-oxa-6-aza-naphthacen-3-ol (6f). White solid, mp: $282-284{ }^{\circ} \mathrm{C}$. IR (KBr): 3482, 3400, 2920, 1627, 1456, 1232, $1177 \mathrm{~cm}^{-1} .{ }^{1} \mathrm{H}$ NMR (300 MHz, DMSO- $\left.d_{6}\right): \delta=1.70\left(\mathrm{~m}, 4 \mathrm{H}, \mathrm{CH}_{2}\right), 2.17\left(\mathrm{~s}, 3 \mathrm{H}, \mathrm{CH}_{3}\right), 2.21\left(\mathrm{~m}, 2 \mathrm{H}, \mathrm{CH}_{2}\right), 2.56$ $\left(\mathrm{m}, 2 \mathrm{H}, \mathrm{CH}_{2}\right), 5.15(\mathrm{~s}, 1 \mathrm{H}, \mathrm{CH}), 5.44\left(\mathrm{~s}, 2 \mathrm{H}, \mathrm{NH}_{2}\right), 6.44-7.12(\mathrm{~m}, 7 \mathrm{H}, \mathrm{ArH}), 9.61(\mathrm{~s}, 1 \mathrm{H}, \mathrm{OH})$. ${ }^{13} \mathrm{C}$ NMR $\left(75 \mathrm{MHz}\right.$, DMSO- $\left.d_{6}\right): \delta=20.4,22.1,22.3,32.0,38.0,98.6,102.5,111.0,111.9,115.9$, $126.9,128.9,129.4,135.2,143.1,151.1,151.2,152.0,155.2,156.8$. Anal. Calcd for $\mathrm{C}_{23} \mathrm{H}_{22} \mathrm{~N}_{2} \mathrm{O}_{2}$ : C, 77.07; H, 6.19; N, 7.82. Found: C, 76.85; H, 6.02; N, 7.65.

11-Amino-12-(3,4,5-trimethoxyphenyl)-7,9,10,12-tetrahydro-8H-5-oxa-6-aza-naphthacen-3ol (6g). White solid, mp: $285-287^{\circ} \mathrm{C}$. IR (KBr): 3468, 3385, 2928, 1630, 1450, 1235, $1180 \mathrm{~cm}^{-1}$. ${ }^{1} \mathrm{H}$ NMR (300 MHz, DMSO-d $\left.d_{6}\right): \delta=1.70\left(\mathrm{~m}, 4 \mathrm{H}, \mathrm{CH}_{2}\right), 2.25\left(\mathrm{~m}, 2 \mathrm{H}, \mathrm{CH}_{2}\right), 2.57\left(\mathrm{~m}, 2 \mathrm{H}, \mathrm{CH}_{2}\right)$, $3.56\left(\mathrm{~s}, 3 \mathrm{H}, \mathrm{CH}_{3}\right), 3.65\left(\mathrm{~s}, 6 \mathrm{H}, \mathrm{CH}_{3}\right), 5.10(\mathrm{~s}, 1 \mathrm{H}, \mathrm{CH}), 5.51\left(\mathrm{~s}, 2 \mathrm{H}, \mathrm{NH}_{2}\right), 6.44-7.00(\mathrm{~m}, 5 \mathrm{H}$, $\mathrm{ArH}), 9.54(\mathrm{~s}, 1 \mathrm{H}, \mathrm{OH}) .{ }^{13} \mathrm{C} \mathrm{NMR}\left(75 \mathrm{MHz}, \mathrm{DMSO}-d_{6}\right): \delta=22.2,22.4,22.9,32.0,55.8,56.0$, 59.8, 98.5, 102.6, 104.6, 111.1, 111.9, 115.7, 129.4, 136.0, 141.9, 151.2, 151.4, 152.2, 152.7 , 155.2, 156.9. Anal. Calcd for $\mathrm{C}_{25} \mathrm{H}_{26} \mathrm{~N}_{2} \mathrm{O}_{5}$ : C, 69.11; H, 6.03; N, 6.45. Found: C, 68.95; H, 5.92; $\mathrm{N}, 6.23$. 
11-Amino-12-(4-dimethylaminophenyl)-7,9,10,12-tetrahydro-8H-5-oxa-6-aza-naphthacen3-ol (6h). Brown solid, mp: $>320^{\circ} \mathrm{C}$. IR (KBr): 3483, 3392, 2922, 1629, 1445, 1240, $1175 \mathrm{~cm}^{-1}$. ${ }^{1} \mathrm{H}$ NMR (300 MHz, DMSO-d $\left.)_{6}\right): \delta=1.70\left(\mathrm{~m}, 4 \mathrm{H}, \mathrm{CH}_{2}\right), 2.23\left(\mathrm{~m}, 2 \mathrm{H}, \mathrm{CH}_{2}\right), 2.51\left(\mathrm{~m}, 2 \mathrm{H}, \mathrm{CH}_{2}\right)$, $2.78\left(\mathrm{~s}, 6 \mathrm{H}, \mathrm{N}\left(\mathrm{CH}_{3}\right)_{2}\right), 5.03(\mathrm{~s}, 1 \mathrm{H}, \mathrm{CH}), 5.37\left(\mathrm{~s}, 2 \mathrm{H}, \mathrm{NH}_{2}\right), 6.43-7.06(\mathrm{~m}, 7 \mathrm{H}, \mathrm{ArH}), 9.61(\mathrm{~s}, 1 \mathrm{H}$, $\mathrm{OH}) .{ }^{13} \mathrm{C}$ NMR $\left(75 \mathrm{MHz}, \mathrm{DMSO}-d_{6}\right): \delta=22.1,22.2,22.4,32.0,37.5,79.1,99.0,102.4,111.0$, $111.8,112.6,116.4,127.6,129.4,134.0,149.0,151.0,151.2,151.8,155.2,156.7$. Anal. Calcd for $\mathrm{C}_{24} \mathrm{H}_{25} \mathrm{~N}_{3} \mathrm{O}_{2}$ : C, 74.39; H, 6.50; N, 10.84. Found: C, 74.25; H, 6.43; N, 10.61.

\section{Acknowledgements}

The authors are thankful to the Department of Biotechnology, New Delhi for financial assistance.

\section{Supplementary Material}

\section{References}

1. (a) Ahmad, S. J. Nat. Prod. 1984, 47, 391. (b) Ulubelen, A. Phytochemistry 1984, 23, 2123. (c) Tantivatana, P.; Ruangrungsi, N.; Vaisiroiroj, V.; Lankin, D. C.; Bhacca, N. S.; Borris, R. P.; Cordell, G. A.; Johnson, L. F. J. Org. Chem. 1983, 48, 268. (d) Munoz, M. A.; Torres, R.; Cassels, B. K. J. Nat. Prod. 1982, 45, 367.

2. (a) Faber, K.; Stueckler, H.; Kappe, T. J. Heterocycl. Chem. 1984, 21, 1171. (b) Johnson, J. V.; Rauckman, S.; Beccanari, P. D.; Roth, B. J. Med. Chem. 1989, 32, 1942. (c) Yamada, N.; Kadowaki, S.; Takahashi, K.; Umeza, K. Biochem. Pharmacol. 1992, 44, 1211.

3. Kolokythas, G.; Pouli, N.; Marakos, P.; Pratsinis, H.; Kletsas, D. Eur. J. Med. Chem. 2006, 41,71

4. Azuine, M. A.; Tokuda, H.; Takayasu, J.; Enjyo, F.; Mukainaka, T.; Konoshima, T.; Nishino, H.; Kapadia, G. J. Pharmacol. Res. 2004, 49, 161.

5. (a) Srivastava, S. K.; Tripathi, R. P.; Ramachandran, R. J. Biol. Chem. 2005, 280, 30273. (b) Brotz-Oesterhelt, H.; Knezevic, I.; Bartel, S.; Lampe, T.; Warnecke- Eberz, U.; Ziegelbauer, K.; Habich, D.; Labischinski, H. J. Biol. Chem. 2003, 278, 39435.

6. Toshiro, S.; Noriko, W. Eur. Pat. Appl. EP 647445 A1 19950412, 1995.

7. Ito, Y.; Kato, H.; Yasuda, S.; Kato, N.; Iwasaki, N.; Nishino, H.; Takeshita, M. Jpn. Kokai Tokkyo Koho, JP 06107664 A2 19940419, 1994.

8. Goto, K.; Yaoka, O; Oe, T. PCT Int. Appl. WO 8401711 A1 19840510, 1984.

9. Maruyama, Y.; Goto, K.; Terasawa, M. Ger. Offen. DE 3010751 19810806, 1981.

10. Ukawa, K.; Ishiguro, T.; Kuriki, H.; Nohara, A. Chem. Pharm. Bull. 1985, 33, 4432. 
11. Curini, M.; Cravotto, G.; Epifano, F.; Giannone, G. Curr. Med. Chem. 2006, 13, 199.

12. O'Kennedy, P.; Thornes, R. D. In Coumarins: Biology, Applications and Mode of Action, John Wiley \& Sons: Chichester, 1997.

13. Ellis, G. P. In The Chemistry of Heterocyclic Compounds. Chromenes, Chromanes, and Chromones; Weissberger, A.; Taylor, E. C. Eds.; John Wiley: New York, 1977; Ch. II, pp $11-139$.

14. (a) Smith, W. P.; Sollis, L. S.; Howes, D. P.; Cherry, C. P.; Starkey, D. I.; Cobley, N. K. J. Med. Chem. 1998, 41, 787. (b) Martinez, A.G.; Marco, L. J. Bioorg. Med. Chem. Lett. 1997, 7,3165 .

15. Kraus, G. A.; Kim, I. J. Org. Chem. 2003, 68, 4517.

16. Hiramoto, K.; Nasuhara, A.; Michiloshi, K.; Kato, T.; Kikugawa, K. Mutat. Res. 1997, 395, 47.

17. Tangmouo, J. G.; Meli, A. L.; Komguem, J.; Kuete, V.; Ngounou, F. N.; Lontsi, D.; Beng, V.P.; Choudhary, M. I.; Sondengam, B. L. Tetrahedron Lett. 2006, 47, 3067.

18. (a) Kitamura, R. O. S.; Romoff, P.; Young, M. C. M.; Kato, M. J.; Lago, J. H. G. Phytochemistry 2006, 67, 2398. (b) Borges, F.; Roleira, F.; Milhazes, N.; Santana, L.; Uriarte, E. Curr. Med. Chem. 2005, 12, 887.

19. (a) Chimenti, F.; Bizzarri, B.; Bolasco, A.; Secci, D.; Chimenti, P.; Carradori, S.; Granese, A.; Rivanera, D.; Lilli, D.; Scaltrito, M. M.; Brenciaglia, M. I. Eur. J. Med. Chem. 2006, 41, 208. (b) Mohr, S. J.; Chirigos, M. A.; Fuhrman, F. S.; Pryor, J. W. Cancer Res. 1975, 35, 3750 .

20. (a) Anderson, D. R.; Hegde, S.; Reinhard, E.; Gomez, L.; Vernier, W. F.; Lee, L.; Liu, S.; Sambandam, A.; Snider, P. A.; Masih, L. Bioorg. Med. Chem. Lett. 2005, 15, 1587. (b) Skommer, J.; Wlodkowic, D.; Matto, M.; Eray, M.; Pelkonen, J. Leukemia Res. 2006, 30, 322.

21. Kulkarni, M. V.; Kulkarni, G. M.; Lin, C. H.; Sun, C. M. Curr. Med. Chem. 2006, 13, 2795.

22. (a) Kemnitzer, W.; Kasibhatla, S.; Jiang, S.; Zhang, H.; Zhao, J.; Jia, S.; Xu, L.;CroganGrundy, C.; Denis, R.; Barriault, N.; Vaillancourt, L.; Charron, S.; Dodd, J.; Attardo, G.; Labrecque, D.; Lamothe, S.; Gourdeau, H.; Tseng, B.; Drewe, J.; Cai, S. X. Bioorg. Med. Chem. Lett. 2005, 15, 4745. (b) Kemnitzer, W.; Drewe, J.; Jiang, S.; Zhang, H.; Wang, Y.; Zhao, J.; Jia, S.; Herich, J.; Labreque, D.; Storer, R.; Meerovitch, K.; Bouffard, D.; Rej, R.; Denis, R.; Blais, C.; Lamothe, S.; Attardo, G.; Gourdeau, H.; Tseng, B.; Kasibhatla, S.; Cai, S. X. J. Med. Chem. 2004, 47, 6299.

23. Dyachenko, V. D.; Chernega, A. N. Russ. J. Org .Chem. 2006, 42, 567.

24. Ballini, R.; Bosica, G.; Conforti, M. L.; Maggi, R.; Mazzacanni, A.; Righi, P.; Sartori, G. Tetrahedron 2001, 57, 1395.

25. Shi, D. Q.; Zhang, S.; Zhuang, Q. Y.; Tu, S. J.; Hu, H. W. Youji Huaxue 2003, 23, 809.

26. Kidwai, M.; Saxena, S.; Rahman Khan, M. K.; Thukral, S. S. Bioorg. Med. Chem. Lett. 2005, $15,4295$.

27. Makarem, S.; Mohammadi, A. A.; Fakhari, A. R. Tetrahedron Lett. 2008, 49, 7194. 
28. (a) Jones, G. In Comprehensive Heterocyclic Chemistry, Katritzky, A. R.; Rees, C. W.; Scriven, E. F. V., Eds.; Pergamon: New York, NY, 1996; Vol. 5, p 167. (b) Cho, C. S.; Oh, B. H.; Kim, T. J.; Shim, S. C. Chem. Commun. 2000, 1885. (c) Jiang, B.; Si, Y. C. J. Org. Chem. 2002, 67, 9449. (d) Mansake, R. H.; Kulka, M. Org. React. 1953, 7, 59. (e) Linderman, R. J.; Kirollos, S. K. Tetrahedron Lett. 1990, 31, 2689. (f) Theclitou, M. E.; Robinson, L. A. Tetrahedron Lett. 2002, 43, 3907.

29. (a) Martinez-Grau, A.; Marco, J. Bioorg. Med. Chem. Lett. 1997, 7, 3165. (b) Li, J. R.; Zhang, L. J.; Yang, X. Q.; Li, Q.; Wang, D.; Wang, C. X.; Shi, D. X.; Zhang, Q. Chin. Chem. Lett. 2008, 19, 15. (c) Selvam, N. P.; Babu, T.H.; Perumal, P. T. Tetrahedron 2009, 65, 8524.

30. (a) de los Rios, C.; Marco, J. L.; Carreiras, M. C.; Chinchon, P. M.; Garcia, A. G.; Villarroya, M. Bioorg. Med. Chem. 2002, 10, 2077. (b) Marco, J. L.; de los Rios, C.; Garcia, A. G.; Villarroya, M.; Carreiras, M. C.; Martins, C.; Eleuterio, A.; Morreale, A.; Orozco, M.; Luque, J. Bioorg. Med. Chem. 2004, 12, 2199. (c) Leon, R.; Marco- Contelles, J.; Garcia, A. G.; Villarroya, M. Bioorg. Med. Chem. 2005, 13, 1167. (d) Marco-Contelles, J.; Leon, R.; de los Rios, C.; Garcia, A. G.; Lopez, M. G.; Villarroya, M. Bioorg. Med. Chem. 2006, 14, 8176. (e) Marco, J. L.; de los Rios, C.; Carreiras, M. C.; Banos, J. E.; Badia, A.; Vivas, N. M. Bioorg. Med. Chem. 2001, 9, 727. (f) Marco-Contelles, J.; Leon, R.; Lopez, M. G.; Garcia, A. G.; Villarroya, M. Eur. J. Med. Chem. 2006, 41, 1464.

31. (a) Zhu, J.; Bienayme, H. Multicomponent Reactions; Wiley-VCH: Weinheim, 2005. (b) Dömling, A. Chem. Rev. 2006, 106, 17. (c) Jimenez-Alonso, S.; Chavez, H.; Estevez-Braan, A.; Ravelo, A.; Feresin, G.; Tapia, A. Tetrahedron 2008, 64, 8938. (d) Tejedor, D.; GarciaTellado, F. Chem. Soc. Rev. 2007, 36, 484. (e) Orru, R. V. A.; de Greef, M. Synthesis 2003, 10, 1471. (f) Weber, L. Drug Discovery Today 2002, 7, 143. (g) Domling, A.; Ugi, I. Angew. Chem. Int. Ed. Engl. 2000, 39, 3168.

32. (a) Loupy, A. Microwaves in Organic Synthesis, 2nd edn. Wiley-VCH: Weinheim, 2006. (b) Caddick, S.; Fitzmaurice, R. Tetrahedron 2009, 65, 3325. (b) Dallinger, D.; Kappe, C. O. Chem. Rev. 2007, 107, 2563. (c) Kappe, C. O. Angew. Chem. Int. Ed. 2004, 43, 6250.

33. (a) Kappe, C. O.; Stadler, A. Microwaves in Organic and Medicinal Chemistry, Wiley-VCH: Weinheim, 2005; pp 182. (b) Shore, G.; Morin, S.; Organ, M. G. Angew. Chem. Int. Ed. 2006, 45, 276. (c) Comer, E.; Organ, M. G. J. Am. Chem. Soc. 2005, 127, 8160.

34. (a) Yavari, I.; Djahaniani, H.; Nasiri, F. Synthesis 2004, 679. (b) Yavari, I.; Djahaniani, H.; Nasiri, F. Tetrahedron. 2003, 59, 9409. (c) Jin, T. S.; Xiao, J. C.; Wang, S. J.; Li, T. S. Ultrason. Sonochem. 2004, 11, 393. (d) Kidwai, M.; Poddar, R. Catal. Lett. 2008, 124, 311. 